\title{
I PARASSITI NELLA STORIA DELL'UOMO: INFEZIONI DEL PASSATO, MALATTIE NEL PRESENTE
}

\author{
CLAUDIO BANDI (*), BEATRICE BISAGLIA (*), ALESSANDRO ALVARO (*)
}

\author{
Nota presentata dal m.e. Enrica Galli \\ (Adunanza del 26 novembre 2020)
}

\begin{abstract}
SuNTO. - Questo articolo presenterà il concetto di "mismatch" evolutivo in relazione alla riduzione nella circolazione di microrganismi e macro-parassiti patogeni (o quasipatogeni) nelle popolazioni umane, nel corso della storia. In particolare, ci riferiremo a quella variegata comunità di parassiti e microrganismi, procarioti o eucarioti, decisamente patogeni, o solo marginalmente od occasionalmente patogeni, che si sono evoluti insieme a noi esseri umani e ai nostri antenati, come abitanti dei nostri corpi. Per semplicità, ci riferiremo a questi organismi indicandoli genericamente come parassiti. Si ritiene che questi organismi, una volta presenti in modo endemico nelle popolazioni della nostra specie, abbiano sviluppato una complessa rete di interazioni con i nostri sistemi fisiologici, in particolare per quanto riguarda la stimolazione del sistema immunitario. In questa dinamica, l'organismo ospite, a sua volta, avrebbe evoluto delle contromisure alla presenza cronica dei parassiti, con una propensione ad una modulazione della risposta immunitaria, in qualche modo specifica per le diverse tipologie di stimolazione determinate dai parassiti. La drastica riduzione nella circolazione dei parassiti nelle società industrializzate avrebbe quindi lasciato alle sue spalle un sistema immunitario sbilanciato. Secondo questa visione, che possiamo indicare come "hygiene hypothesis" (ipotesi dell'igiene), l'aumento dell'incidenza di varie patologie immuno-mediate nei paesi industrializzati sarebbe attribuibile, almeno in parte, a questo sbilanciamento della funzione immunitaria.
\end{abstract}

$$
* * *
$$

ABSTRACT. - This article will present the concept of evolutionary mismatch, in relation to the reduction in the circulation of pathogenic (or quasi-pathogenic) microorganisms

(") Dipartimento di Bioscienze e CRC Pediatrico Romeo ed Enrica Invernizzi, Università degli Studi di Milano, Italy. E-mail: claudio.bandi@unimi.it 
and macro-parasites in human populations, throughout human and pre-human history. In particular, we will refer to the variegated community of parasites and microorganisms, prokaryotes or eukaryotes, pathogenic or only marginally or occasionally pathogenic, which evolved as inhabitants of our bodies together with us human beings and our ancestors. For simplicity, we will generically refer to these organisms as parasites. These organisms, once present endemically in the populations of our species, are believed to have developed a complex network of interactions with our physiological systems, particularly with regard to stimulation of the immune system. In this dynamic, the host organism, in turn, would have evolved countermeasures to the chronic presence of parasites, with a bias towards the modulation of the immune response, somehow specific for the different types of stimulation determined by the parasites. The drastic reduction in the circulation of parasites in industrialized societies would therefore have left an imbalanced immune system behind it. According to this view, which we can refer to as the "hygiene hypothesis", the increase in the incidence of various immune-mediated diseases in industrialized countries would be attributable, at least in part, to this imbalance of the immune function.

\section{INTRODUZIONE: DISADATTAMENTO EVOLUTIVO E IPOTESI DELL'IGIENE}

Un concetto chiave nella medicina evoluzionistica è quello del disadattamento, o "mismatch", evolutivo [1]. Secondo questo concetto, una specie evolutasi in un determinato ambiente potrebbe non essere adattata a un ambiente nuovo o diverso. In altre parole, una discrepanza nelle condizioni in cui un organismo vive oggi rispetto alle condizioni in cui i suoi geni (e quindi il suo corpo) vennero modellati dalla selezione naturale, potrebbe implicare un disadattamento in diversi tratti ereditari; questo, a sua volta, potrebbe portare a perturbazioni nell'equilibrio omeostatico complessivo dell'organismo. In questo contesto, dovremmo considerare lo scenario in cui si suppone si sia evoluto Homo sapiens. Che fossimo principalmente cacciatori o raccoglitori, le condizioni igieniche dei nostri antenati nell'era pre-agricola erano probabilmente simili a quelle di molti animali selvatici. Dobbiamo considerare che l'essere infettati ripetutamente e/o cronicamente da parassiti rappresenta la "normalità" per la maggior parte dei mammiferi selvatici [2]. Non c'è motivo di pensare che così non fosse per i nostri antenati, che si evolvettero in piccole comunità nomadi formate da 150-250 individui per decine di migliaia di anni sino all'origine dell'agricoltura e dell'allevamento [3]. La storia dell'umanità (o di parte di essa) andò quindi incontro ad un cambiamento radicale con il passaggio all'agricoltura e 
all'allevamento, in un periodo compreso tra circa 10.000 e 5000 anni fa - a seconda dei luoghi [4]. A seguito di questo cambiamento, la coesistenza dell'uomo con i parassiti non solo non sarebbe diminuita, ma sarebbe addirittura aumentata, almeno per alcuni patogeni. La condizione di semi-convivenza con gli animali (in relazione all'allevamento) e lo stile di vita sedentario (legato alle pratiche agricole) avrebbero infatti facilitato la trasmissione di agenti patogeni dal bestiame all'uomo e la trasmissione oro-fecale dei parassiti. La coesistenza con i parassiti e con una varietà di altri microrganismi "quasi patogeni" è quindi da considerarsi come la condizione normale per i nostri antenati nell'era preagricola. In altre parole, noi e i nostri antenati ci siamo evoluti ospitando nei nostri corpi diversi parassiti, oltre ad una comunità di microrganismi costituenti il microbiota, che doveva essere verosimilmente molto "variegato". Questo probabilmente ha portato alla creazione di una complessa rete di interdipendenza tra noi, i nostri parassiti e tutti i microbi che abbiamo ospitato come specie durante la nostra storia. Poi è successo qualcosa. Con il passaggio alla società industriale, e successivamente con l'adozione di condizioni di vita più igieniche, l'ambiente umano ha subito un rapido e importante cambiamento [5]. Anche la dieta è cambiata nel tempo, prima con il passaggio all'agricoltura, poi con i progressi nella produzione e distribuzione alimentare nel XIX e XX secolo [6] [7]. In conclusione, noi, esseri umani, ci siamo evoluti in un tipo di ambiente che non esiste più, e ora viviamo in una condizione radicalmente diversa: stiamo verosimilmente vivendo un una condizione di disadattamento evolutivo.

In sintesi, il nostro sistema immunitario si è evoluto, per un lungo periodo di tempo, per permetterci non solo di eliminare determinati agenti patogeni, ma anche per permetterci di tollerare la presenza di una serie di altri organismi patogeni o quasi patogeni, che sono stati indicati come i "dirty old friends" (vecchi sporchi amici) [8]. E piuttosto ovvio che un equilibrio omeostatico che è stato modellato per essere mantenuto in presenza di un pesante carico di fattori immunomodulanti verrà alterato dopo la scomparsa di una parte di questi fattori; i vecchi sporchi amici erano sicuramente un pesante fardello di fattori immunomodulanti.

Il concetto del disadattamento evolutivo, in relazione nello specifico alla riduzione della prevalenza dei "dirty old friends", costituisce il background teorico per la "hygiene hypothesis" (ipotesi dell'igiene). Proposta da Strachan nel 1989, la "hygiene hypothesis" prevede che 
l'aumento dell'incidenza di malattie infiammatorie e autoimmuni nel corso del XX secolo sia stato causato dalla ridotta esposizione ai parassiti e ad una varietà microrganismi quasi-patogeni o commensali nei paesi industrializzati [9] [10] [11]. Tra queste patologie, le malattie infiammatorie intestinali ("Inflammatory Bowel Diseases" - IBD) occupano una posizione di rilievo, verosimilmente in relazione alla stretta interazione tra le comunità microbiche (ed eventualmente parassitarie) presenti nell'intestino e la risposta immune, e quindi eventualmente infiammatoria, a livello della mucosa intestinale. Inoltre, sempre tra le patologie che hanno aumentato la propria prevalenza nel corso della seconda metà del XX Secolo, in parallelo alla riduzione nell'incidenza di diverse malattie infettive, possiamo ricordare il diabete di tipo 1, diverse forme di allergia e le patologie neuroinfiammatorie.

\section{DALla "HYGIENE HYPOTHESIS" ALLA RICERCA DI MOLECOLE AD AZIONE IMMUNOMODULANTE ASSOCIATE AI PARASSITI}

La "hygiene hypothesis" è, per definizione, un'ipotesi. Risulta supportata dai risultati di molte indagini epidemiologiche ed è coerente con le attuali conoscenze sulla risposta immunopatologica, nelle sue diverse forme, così come con quanto noto circa le azioni pro-infiammatorie o immunomodulanti innescate dai parassiti. Essendo stati pubblicati numerosi articoli su questi argomenti (e.g. [10] [11]) non intendiamo occuparcene in questa sede. Preferiamo invece presentare un esempio relativo ad uno studio ispirato dai concetti della "hygiene hypothesis" e del disadattamento evolutivo, che ha portato all'identificazione di una proteina associata a parassiti dell'uomo e degli animali, potenzialmente coinvolta nella modulazione della risposta immunitaria e nella protezione dell'ospite nei confronti di altre patologie.

Lo studio a cui ci riferiamo, di Varotto-Boccazzi e collaboratori [12], riguarda due parassiti: Dirofiliaria immitis (Leidy, 1856), un verme cilindrico parassita del cane, e Leishmania infantum Nicolle, 1908, un protozoo flagellato, anch'essa parassita del cane, ma anche, seppur non frequentemente, dell'uomo. Nel corso degli ultimi trent'anni, nella Pianura Padana si è riscontrata una progressiva riduzione della circolazione di $D$. immitis, in particolare nei cani trattati per la prevenzione della dirofilariosi, la malattia di cui il nematode è l'agente eziologico (virtualmente tutti i cani di proprietà vengono trattati, i.e. la stragrande maggio- 
ranza dei cani in quest'area geografica). In parallelo, L. infantum, una volta sconosciuta in Pianura Padana, ha iniziato a fare la sua comparsa con focolai sempre più estesi. E' stato proposto che L. infantum abbia ampliato il proprio areale geografico per ragioni connesse con il fenomeno del riscaldamento globale, che favorirebbe l'ampliamento dell'areale dei flebotomi, gli insetti che trasmettono le leishmanie. Tuttavia, come sottolineato da Varotto-Boccazzi e collaboratori, alcune ricerche suggeriscono che le filarie potrebbero effettivamente interferire con la leishmaniosi, proteggendo il cane da questa malattia [13] [14].

Queste osservazioni hanno portato Varotto-Boccazzi e collaboratori a porsi le domande alla base del loro studio: è possibile che molecole derivate da una filaria possano proteggere un animale dall'infezione da parte di una leishmania? Ed è quindi possibile che la riduzione nella presenza di filarie nel cane in Pianura Padana abbia favorito la diffusione di leishmania? Considerando gli aspetti evoluzionistici, queste domande sono assolutamente ragionevoli. Una filaria come quella del cane può sopravvivere nel suo ospite per anni; proteggendo il cane da infezioni come la leishmaniosi, la filaria protegge sé stessa, allungando la sua vita e aumentando la sua generazione di progenie. Insomma, è ragionevole assumere che nel corso dell'evoluzione ci sia stato un premio selettivo per le filarie per determinare effetti protettivi sull'ospite nei confronti di altri agenti infettivi. E' interessante notare, inoltre, che studi sulle filarie di roditori suggeriscono che questi vermi potrebbero proteggere l'ospite anche nei confronti delle sepsi batteriche [15].

Tornando allo studio condotto da Varotto-Boccazzi e collaboratori, la prima fase della ricerca è consistita nella scelta di una molecola associata alla filaria del cane che potesse essere in grado di interferire con l'infezione da parte di Leishmania. Questa molecola è stata identificata in una proteina nota come "Wolbachia Surface Protein" (WSP). Si tratta una molecola prodotta non direttamente dalla filaria, ma da Wolbachia, un batterio simbionte stabilmente associato con le filarie; in ogni caso, una molecola sempre presente nella filaria del cane, e potenzialmente in grado di modulare la risposta immunitaria in modo da proteggere l'animale da infezioni come quella da leishmania. Una volta identificata la proteina WSP come agente immunomodulante potenzialmente protettivo nei confronti di determinate infezioni, lo studio è proseguito con esperimenti in vitro, volti a determinare la capacità di WSP di proteggere cellule del sistema immunitario dall'infezione da parte di leishmania. Nello specifico, sono state utilizzate cellule macro- 
fagiche di mammifero altamente suscettibili all'infezione da parte di leishmania: cellule al cui interno questo parassita si moltiplica in modo efficace. Esponendo queste cellule a WSP si osservava una loro forte attivazione, con inibizione della crescita di leishmania e sua degenerazione, con esiti protettivi in parte sovrapponibili a quelli determinati dall'Amfotericina B, un farmaco di scelta per la cura della leishmaniosi (lo studio in effetti prevedeva la somministrazione di WSP alle cellule attraverso la sua produzione da parte del batterio Asaia, utilizzato come piattaforma di espressione e veicolo vaccinale; si tratta di dettagli a cui il lettore interessato potrà accedere attraverso la lettura dell'articolo di Varotto-Boccazzi et al. 2020 [11] e di Epis et al. 2020 [16]).

In sintesi, a partire dall'ipotesi che una riduzione della prevalenza della filariosi del cane in Pianura Padana abbia favorito la diffusione di leishmania, è stato condotto uno studio che ha permesso di identificare una molecola associata alla filaria in grado di interferire con la crescita intracellulare di leishmania. Quindi, uno studio coerente con la "hygiene hypothesis", ma anche con potenzialità applicativa, in relazione alla possibilità che la proteina saggiata dagli autori possa essere sviluppata come agente immunomodulante per la prevenzione o la cura della leishmaniosi.

\section{CONCLUSIONI}

La "hygiene hypothesis" ha contribuito allo sviluppo della medicina evolutiva (o darwiniana), una sotto-disciplina della biologia evoluzionistica che si pone l'obiettivo di identificare alterazioni patologiche che possano essere interpretate sulla base di fenomeni evolutivi. Come già sottolineato, uno dei concetti chiave della medicina darwiniana è quello del disadattamento evolutivo (vedi introduzione). Una trattazione circa la validità della "hygiene hypothesis" non rientrava tra gli scopi di questo articolo. Piuttosto, una volta presentato il concetto, abbiamo voluto enfatizzare con un esempio, come la "hygiene hypothesis" abbia stimolato la ricerca. In conclusione, ci sentiamo di affermare che:

- La "hygiene hypothesis" ha portato alla formulazione di nuove interpretazioni e quesiti circa le malattie a base immunopatologica, sia in relazione ai meccanismi di sviluppo di tali patologie, sia in relazione alla loro epidemiologia;

- Le indagini sul microbiota sono ancora influenzate dalla "hygiene hypothesis"; 
- L'ipotesi ha portato ad un rinnovato interesse per le malattie parassitarie e ha stimolato lo studio dei meccanismi che i parassiti utilizzano per modulare la risposta immunitaria dell'ospite;

- Lo studio dei parassiti ha portato all'identificazione di molecole coinvolte nella stimolazione e nella modulazione della risposta immune, molecole che si presentano come potenzialmente sviluppabili per possibili applicazioni profilattiche e terapeutiche.

\section{RINGRAZIAMENTI}

Le ricerche di Claudio Bandi sono in parte sostenute dalla Fondazione Romeo ed Enrica Invernizzi.

\section{REFERENZE}

[1] P.D. Gluckman, M.A. Hanson, F.M. Low, Evolutionary and developmental mismatches are consequences of adaptive developmental plasticity in bumans and have implications for later disease risk, Philosophical Transactions of the Royal Society of London B Biological Sciences 374, 1770 (2019), 20180109.

[2] F. Bordes, S. Morand, The impact of multiple infections on wild animal hosts: a review, Infection Ecology \& Epidemiology 1 (2011), 7346.

[3] D. Lieberman, The Story of the Human Body: Evolution, Health, and Disease, Pantheon Books, 2013.

[4] J.M. Diamond, Guns, Germs, and Steel: The Fates of Human Societies, W.W. Norton, 1997.

[5] G. Fields, Urbanization and the Transition from Agrarian to Industrial Society, Berkeley Planning Journal 13, 1 (1999).

[6] A.N. Crittenden, S.L. Schnorr, Current views on bunter-gatherer nutrition and the evolution of the buman diet, American Journal of Physical Anthropology 162 Supplement 63 (2017), 84-109.

[7] W.P.T. James, R.J. Johnson, J.R. Speakman, D.C. Wallace, G. Frühbeck, P.O. Iversen, P.J. Stover, Nutrition and its role in buman evolution, Journal of Internal Medicine 285, 5 (2019), 533-549.

[8] G.A. Rook, 99th Dablem conference on infection, inflammation and chronic inflammatory disorders: darwinian medicine and the 'bygiene' or 'old friends' bypothesis, Clinical \& Experimental Immunology 160, 1 (2010), 70-79. 
[9] D.P. Strachan, Hay fever, bygiene, and housebold size, British Medical Journal 299, 6710 (1989), 1259-1260.

[10] J.-F. Bach, The bygiene bypothesis in autoimmunity: the role of pathogens and commensals, Nature Reviews Immunology 18, 2 (2018), 105-120.

[11] S. Panelli, S. Epis, L. Cococcioni, M. Perini, M. Paroni, C. Bandi, L. Drago, G.V. Zuccotti, Inflammatory bowel diseases, the hygiene bypothesis and the other side of the microbiota: Parasites and fungi, Pharmacological Research 159 (2020), 104962.

[12] I. Varotto-Boccazzi, S. Epis, Arnoldi I., Y. Corbett, P. Gabrieli, M. Paroni, R. Nodari, N. Basilico, L. Sacchi, M. Gramiccia, L. Gradoni, V. Tranquillo, C. Bandi, Boosting immunity to treat parasitic infections: Asaia bacteria expressing a protein from Wolbachia determine M1 macrophage activation and killing of Leishmania protozoans, Pharmacological Research 161 (2020), 105288.

[13] C. Maia, L. Altet, L. Serrano, J.M. Cristóvão, M. Dolores Tabar, O. Francino, L. Cardoso, L. Campino, X. Roura, Molecular detection of Leishmania infantum, filariae and Wolbachia spp. in dogs from southern Portugal, Parasites Vectors 9, 1 (2016), 170.

[14] P.K. Murthy, S. Dixit, R.L. Gaur, R. Kumar, M.K. Sahoo, N. Shakya, S.K. Joseph, S. Palne, S. Gupta, Influence of Brugia malayi life stages and BmAFII fraction on experimental Leishmania donovani infection in hamsters, Acta Tropica 106, 2 (2008), 81-89.

[15] F. Gondorf, A. Berbudi, B.C. Buerfent, J. Ajendra, D. Bloemker, S. Specht, D. Schmidt, A.-L. Neumann, L.E. Layland, A. Hoerauf, M.P. Hübner, Chronic filarial infection provides protection against bacterial sepsis by functionally reprogramming macrophages, PLOS Pathogens 11, 1 (2015), e1004616.

[16] S. Epis, I. Varotto-Boccazzi, E. Crotti, C. Damiani, L. Giovati, M. Mandrioli, M. Biggiogera, P. Gabrieli, M. Genchi, L. Polonelli, D. Daffonchio, G. Favia, C. Bandi, Chimeric symbionts expressing a Wolbachia protein stimulate mosquito immunity and inbibit filarial parasite development, Communications Biology 3, 105, (2020), 10.1038/s42003-020-0835-2 